\title{
HIV-1 Group P is unable to antagonize human tetherin by Vpu, Env or Nef
}

\author{
Daniel Sauter ${ }^{1}$, Stéphane Hué ${ }^{2}$, Sarah J Petit ${ }^{2}$, Jean-Christophe Plantier ${ }^{3}$, Greg J Towers ${ }^{2}$, Frank Kirchhoff ${ }^{*}$ and \\ Ravindra K Gupta ${ }^{2 *}$
}

\begin{abstract}
Background: A new subgroup of HIV-1, designated Group P, was recently detected in two unrelated patients of Cameroonian origin. HIV-1 Group P phylogenetically clusters with SIVgor suggesting that it is the result of a crossspecies transmission from gorillas. Until today, HIV-1 Group P has only been detected in two patients, and its degree of adaptation to the human host is largely unknown. Previous data have shown that pandemic HIV-1 Group $\mathrm{M}$, but not non-pandemic Group O or rare Group N viruses, efficiently antagonize the human orthologue of the restriction factor tetherin (BST-2, HM1.24, CD317) suggesting that primate lentiviruses may have to gain antitetherin activity for efficient spread in the human population. Thus far, three SIV/HIV gene products ( $v p u$, nef and env) are known to have the potential to counteract primate tetherin proteins, often in a species-specific manner. Here, we examined how long Group P may have been circulating in humans and determined its capability to antagonize human tetherin as an indicator of adaptation to humans.

Results: Our data suggest that HIV-1 Group P entered the human population between 1845 and 1989. Vpu, Env and Nef proteins from both Group P viruses failed to counteract human or gorilla tetherin to promote efficient release of HIV-1 virions, although both Group P Nef proteins moderately downmodulated gorilla tetherin from the cell surface. Notably, Vpu, Env and Nef alleles from the two HIV-1 P strains were all able to reduce CD4 cell surface expression.

Conclusions: Our analyses of the two reported HIV-1 Group P viruses suggest that zoonosis occurred in the last 170 years and further support that pandemic HIV-1 Group M strains are better adapted to humans than nonpandemic or rare Group O, N and P viruses. The inability to antagonize human tetherin may potentially explain the limited spread of HIV-1 Group P in the human population.
\end{abstract}

\section{Background}

The determinants of successful cross-species transmissions of primate lentiviruses are poorly understood, and may be critical in developing successful preventive strategies against primate lentiviral zoonoses in the future. Besides environmental factors, the adaptation of the virus to the immune system of its new host may determine its spread after zoonotic transmission. Mammalian restriction factors are interferon-inducible members of the innate immune system, and include the proteins TRIM $5 \alpha$ and APO$\mathrm{BEC} 3 \mathrm{G} / \mathrm{F}$ [1-3]. These proteins evolved under positive

\footnotetext{
* Correspondence: frank.kirchhoff@uni-ulm.de; rebmrag@ucl.ac.uk ${ }^{1}$ Institute of Molecular Virology, Ulm University Medical Center, 89069, UIm, Germany

${ }^{2}$ Division of Infection and Immunity, University College London, Gower Street, London, WC1E 6BT, UK

Full list of author information is available at the end of the article
}

selection pressure and provide barriers to cross-species transmission. The high variability of primate lentiviruses, however, may allow them to become resistant to TRIM $5 \alpha$ through capsid sequence variation [4], and to gain activity against the restriction factors in the new host by the acquisition of mutations in their accessory genes. Tetherin, the most recently described restriction factor expressed by all major cellular targets of HIV infection (including CD4+ lymphocytes), is a transmembrane protein found at the plasma membrane, with an extracellular domain bearing a GPI anchor at its $\mathrm{C}$-terminus [5]. This unusual topology with two membrane anchors enables tetherin to tether budding viruses to the infected cell, thus preventing their release, with subsequent internalization and degradation [6-9]. The delicate balance between antiviral restriction factors and viral escape is highlighted by the fact that
C Biomed Central

(C) 2011 Sauter et al; licensee BioMed Central Ltd. This is an Open Access article distributed under the terms of the Creative Commons Attribution License (http://creativecommons.org/licenses/by/2.0), which permits unrestricted use, distribution, and reproduction in any medium, provided the original work is properly cited. 
single amino acid changes in viral or mammalian proteins may lead to gain or loss of function [10-13].

Most simian immunodeficiency viruses (SIVs), including SIVcpz and SIVgor that are found in chimpanzees and gorillas (reviewed in [14]), and represent the direct precursors of HIV-1, use Nef to antagonize tetherin [15-20], whilst SIV infecting certain monkeys (SIVgsn, SIVmus, SIVmon) use Vpu $[17,21]$. Human tetherin, however, is resistant to Nef due to a deletion in its cytoplasmic tail [15-20]. During its adaptation to humans, HIV-1 Group M has perfectly mastered this hurdle by switching from Nef to Vpu to antagonize human tetherin $[16,17]$. In contrast, $\mathrm{Vpu}$ proteins from non-pandemic Group $\mathrm{O}$ viruses do not antagonize tetherin and those of the rare Group $\mathrm{N}$ viruses gained some modest antitetherin activity but do not degrade CD4 $[17,21]$. The finding that pandemic HIV-1 M strains counteract human tetherin substantially more effectively than nonpandemic or rare Group $\mathrm{O}$ and $\mathrm{N}$ viruses suggests that effective tetherin antagonism may have been required for the efficient global spread of HIV/AIDS. The recent identification of a fourth group of HIV-1, designated HIV-1 Group P, that is closely related to SIVgor and most likely resulted from an independent gorilla-human transmission $[22,23]$, provides another interesting opportunity to evaluate a possible correlation between effective tetherin antagonism and viral spread. Thus far, only two HIV-1 Group P strains have been described. The first one (RBF168) was isolated from a woman who had lived near Yaoundé, Cameroon [22]. The second (06CMU14788) was identified in a stored serum sample from a male patient admitted to Yaoundé hospital in 2006 [23]. In the present study, we performed phylogenetic analyses to assess how long Group P viruses may have been circulating in the human population and examined whether lack of anti-tetherin activity and thus suboptimal adaptation to humans may explain their limited spread in the human population.

\section{Results}

HIV-1 Group P entered the human population between 1845 and 1989

To determine whether HIV-1 Group P may have a relatively recent origin, we estimated the likely date of its zoonotic transmission by dating the HIV-1 Group P/SIVgor time of most recent common ancestor (tMRCA), as well as the MRCA of RBF168 and 06CMU14788. This analysis was performed using the Bayesian Markov chain Monte Carlo (MCMC) framework [24] implemented in the package BEASTv1.5.2. [25], using gag, pol and env gene sequences. We found good agreement in the HIV-1 Group P/SIVgor tMRCA estimates (Figure 1), obtained on the basis of the gag (1899 [95\% Higher Probability Density: 1855-1938]), pol (1864 [1813-1912]) and env genes (1845 [1766-1912]). We also assessed the impact of including partial gag and pol sequences, of 1056 and 891 nucleotides respectively, from a more divergent SIVgor strain (BQ664) for which full-length sequence is not available [26]. No significant changes in the HIV-1 Group P/SIVgor tMRCA estimates were observed (data not shown). The tMRCA for RBF168 and 06CMU14788 was also estimated at each of the three loci (Figure 1), and gave consistent results as follows: gag 1982 (19701992), pol 1989 (1980-1995) and env 1971 (1951-1988).

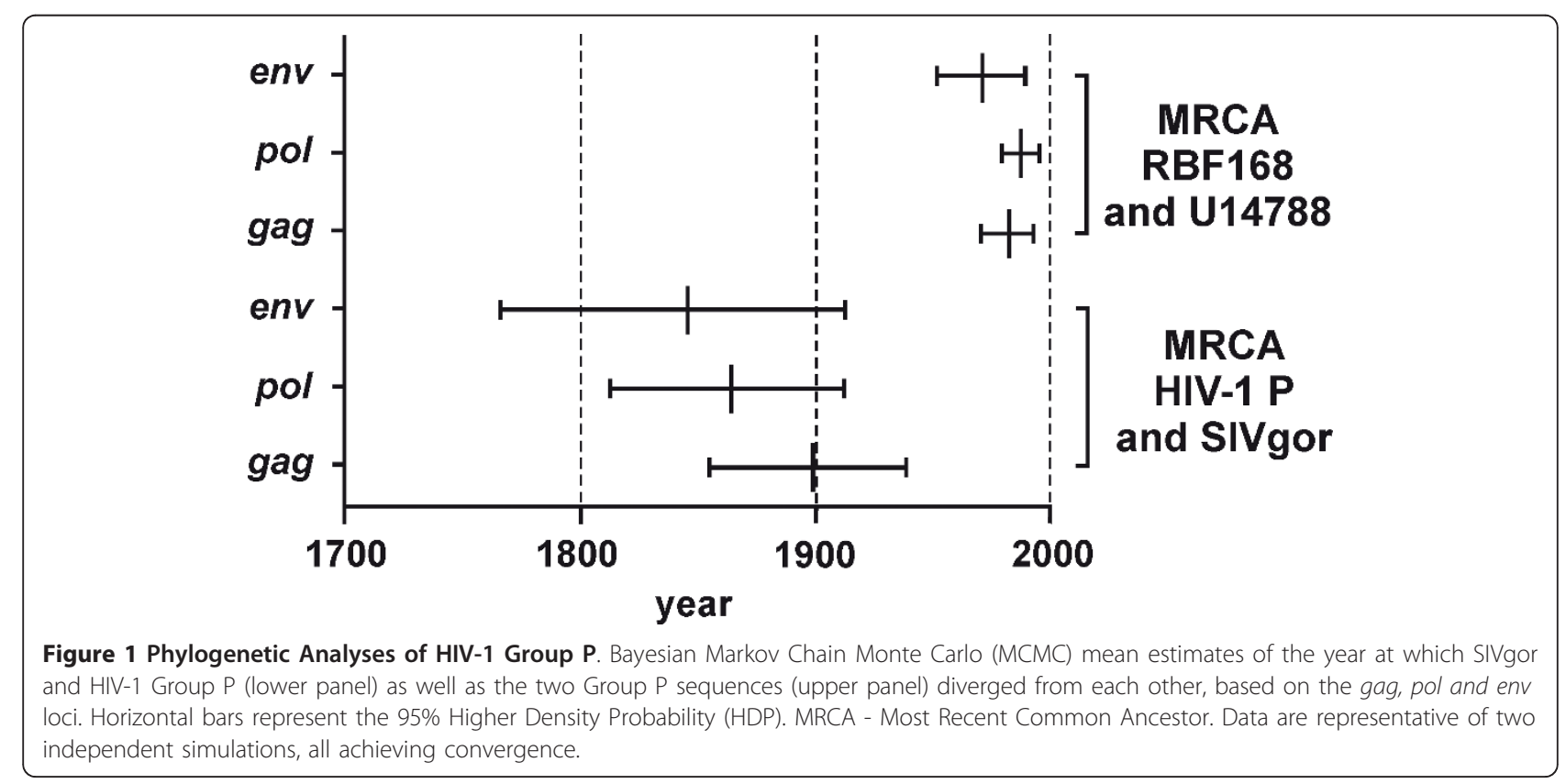


Based on these analyses, we estimate that zoonosis occurred between 1845 and 1989. Sequences from additional HIV-1 Group P viruses and SIVgor strains would be required for more precise estimates. HIV-1 Group M is thought to have entered the human population around 1908 (1884-1924), Group O between 1905 and 1920 (1866-1940) [27,28], and Group N around 1963 (19481977) $[28,29]$. Thus, the four independent transmission events that gave rise to HIV-1 Groups $\mathrm{M}, \mathrm{O}, \mathrm{N}$ and $\mathrm{P}$ most likely all occurred relatively recently, i.e. within the last 170 years.

\section{HIV-1 P Group Vpu and Nef downregulate CD4 cell surface expression}

We next assessed the ability of the two HIV-1 Group P viruses to antagonize gorilla and human tetherin as an indicator of adaptation to humans. To this end, the $v p u$ or nef genes from HIV-1 P RBF168 and 06CMU14788 as well as two SIVgor strains (cp2139 and BQ644) were cloned into the bi-cistronic CMV-based pCGCG expression vector coexpressing the enhanced version of green fluorescent protein (eGFP) [30] and fused to a C-terminal AU1-tag. As further controls, we included nef and $v p u$ alleles from five divergent HIV-1 $\mathrm{M}$ strains representing subtypes B (NL4-3, JR-CSF, founder virus CH106), C (C.KA) and D (D.ZA). Western blot analyses showed that all $\mathrm{Vpu}$ and Nef proteins were expressed at detectable levels (Figure 2A, B). Of note, our blots are consistent with previous observations that some $\mathrm{Vpu}$ proteins tend to aggregate and that the migration pattern on Western blots does not always reflect the calculated molecular weight [17]. Next, we determined whether the HIV-1 P Vpu and Nef proteins reduce cell surface expression of CD4. To measure this, 293T cells were cotransfected with vectors coexpressing $v p u$ or nef and eGFP (or eGFP alone for control) together with a human CD4 expression construct, as previously described $[17,31,32]$. In the absence of Vpu or Nef, the cells expressed high levels of CD4 (Figure 2C). However, coexpression of the HIV-1 P Vpu decreased CD4 surface expression by more than $90 \%$ and HIV-1 P Nef reduced CD4 levels by 55 to 75\% (Figure 2D).

\section{HIV-1 P Group Vpu and Nef are unable to counteract tetherin}

We next tested the activity of Group P Vpu and Nef proteins against human and gorilla tetherin. To this end, we determined the levels of tetherin surface expression and infectious virus yields from HEK 293T cells cotransfected with a vpu/nef-deleted ( $\Delta v p u \Delta n e f)$ HIV-1 Group M NL43 proviral construct [33], a tetherin expression plasmid, and a vector expressing Vpu or Nef [17]. As expected, HIV-1 Group M Vpus downregulated human tetherin about 5- to 6-fold (Figure 3A, B). In contrast, both HIV-1 Group P vpu alleles did not reduce tetherin cell surface expression (Figure 3B). This is not surprising since HIV-1 Group P Vpus lack the AxxxAxxxW transmembrane motif known to be critical for tetherin antagonism by HIV-1 Group M Vpus and may thus not interact with this restriction factor [34] (Additional file 1). Expression of HIV-1 P RBF168 and 06CMU14788 Nef did not affect surface expression of human tetherin either, but slightly decreased gorilla tetherin surface expression by 1.5 - to 2.5-fold (Figure 3B). Unlike the SIVgor Nef, however, both HIV-1 Group P Nef proteins were unable to increase virion release in the presence of gorilla tetherin (Figure 3C-E and Additional file 2). This observation is consistent with reports that Vpu-mediated downmodulation of tetherin and viral release may be separable activities $[35,36]$, although a similar finding has not yet been reported for Nef. Importantly, neither HIV-1 Group P Vpu nor Nef proteins were able to increase infectious virion or p24 release in the presence of human tetherin (Figure 3C-E and S2). This lack of anti-tetherin activity of Nef and Vpu shows that Group P viruses are not yet optimally adapted to the human host.

\section{Group P Envs reduce CD4 cell surface expression but do not counteract tetherin}

Besides Nef and Vpu, Env is the third primate lentiviral protein known to display anti-tetherin activity $[11,37]$. Thus, we also assessed the ability of HIV-1 Group P Env to antagonize tetherin. For this, both HIV-1 Group P env genes were chemically synthesized and cloned into the pCAGGS expression vector. Functional expression was assessed by measuring the levels of CD4 on 293T cells cotransfected with CD4 and Env expression constructs. We found that Group P Envs reduced CD4 surface expression levels about 2-fold, which is similar to the Group M NL4-3 Env (Figure 4A). We further demonstrated expression of functional RBF168 and 06CMU14788 env gene products by successful pseudotyping of an env-deficient HIV-1 M NL4-3 proviral construct and subsequent infection of TZM-bl reporter cells (Figure 4B).

We then tested antagonism of human tetherin by cotransfecting a vpu/nef/env-deleted (DvpuDnefDenv) HIV-1 NL4-3 proviral construct along with RBF168 and 06CMU14788 Env in the presence of increasing amounts of tetherin expression plasmid. Both HIV-1 Group P Envs were unable to downregulate human tetherin from the cell surface (Figure $4 \mathrm{C}$ ) and failed to increase virion release in the presence of human tetherin (Figure 4D). Since a previous study has shown that HIV2 Env only antagonizes endogenous tetherin in Hela cells, but not transiently expressed tetherin in HEK 293T cells [37], we transfected Env expression plasmids along with the DvpuDnefDenv HIV-1 NL4-3 proviral construct in HeLa cells and still found no effect of Group P Env on viral release (data not shown). 


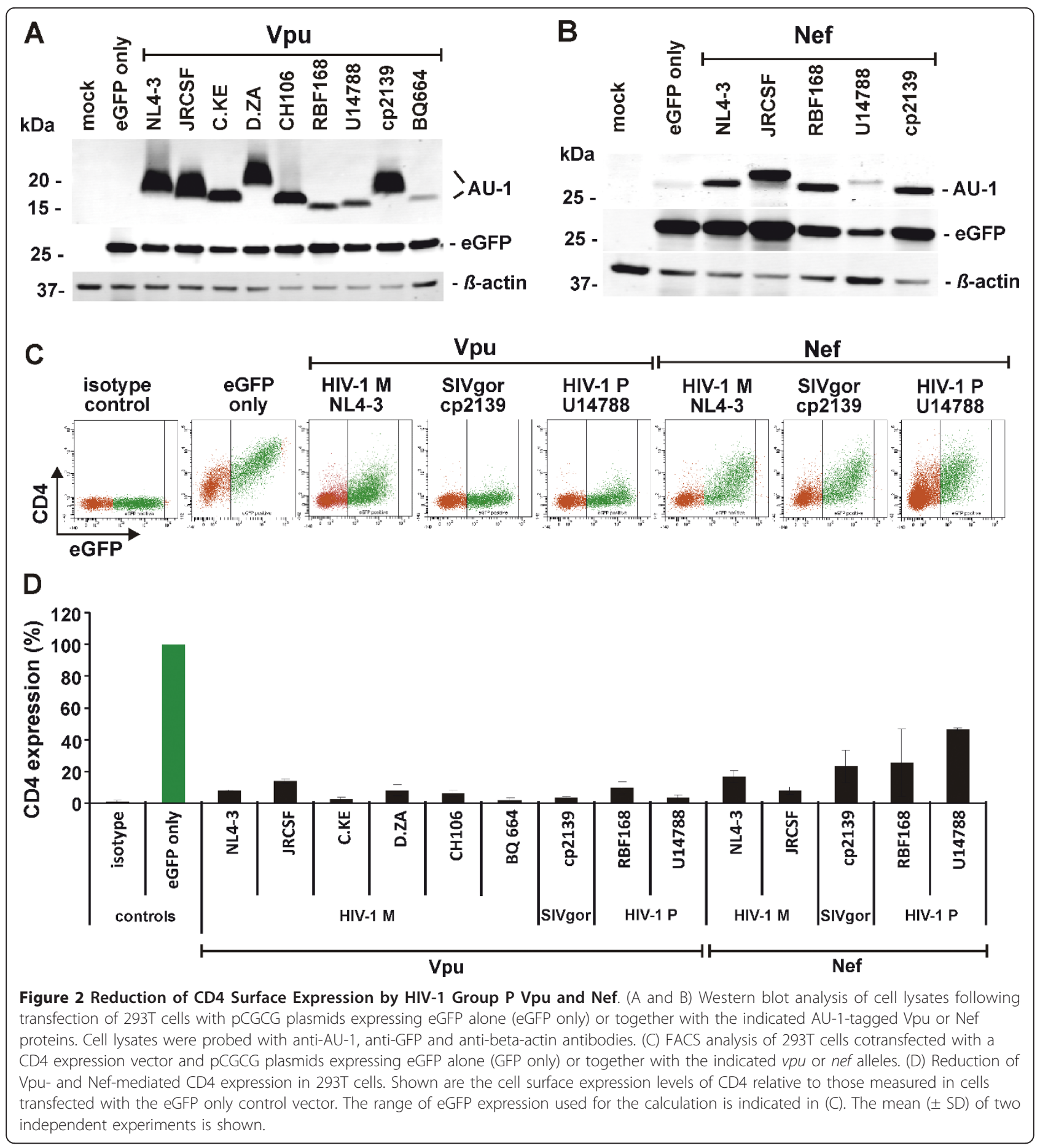

\section{Discussion}

Previous studies suggest that effective tetherin antagonism may have been a prerequisite for the effective global spread of HIV/AIDS [16,17]. The human tetherin orthologue contains a deletion in the N-terminal cytoplasmic tail that renders human tetherin resistant to the accessory viral Nef protein. Nef is used by most primate lentiviruses, including SIVcpz, SIVgor and SIVsmm, the direct precursors of HIV-1 and HIV-2, to counteract tetherin in their respective host species. Thus, tetherin represents a significant barrier to effective zoonotic transmission of primate lentiviruses [15-20]. Pandemic HIV-1 Group M strains fully cleared this hurdle by switching from Nef to Vpu to counteract tetherin in the new human host [17]. In contrast, the non-pandemic Group $\mathrm{O}$ viruses seem to be unable to antagonize 


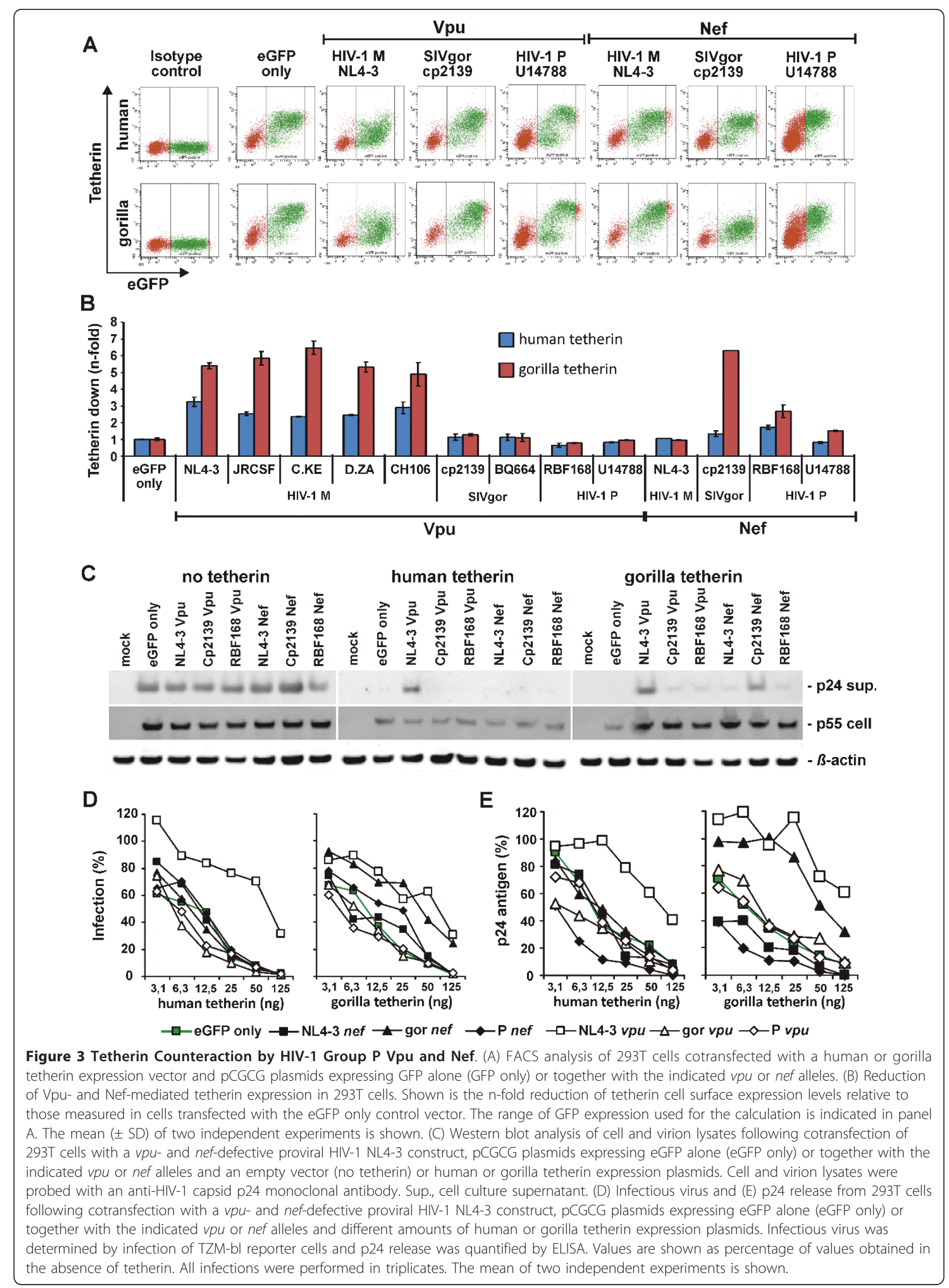



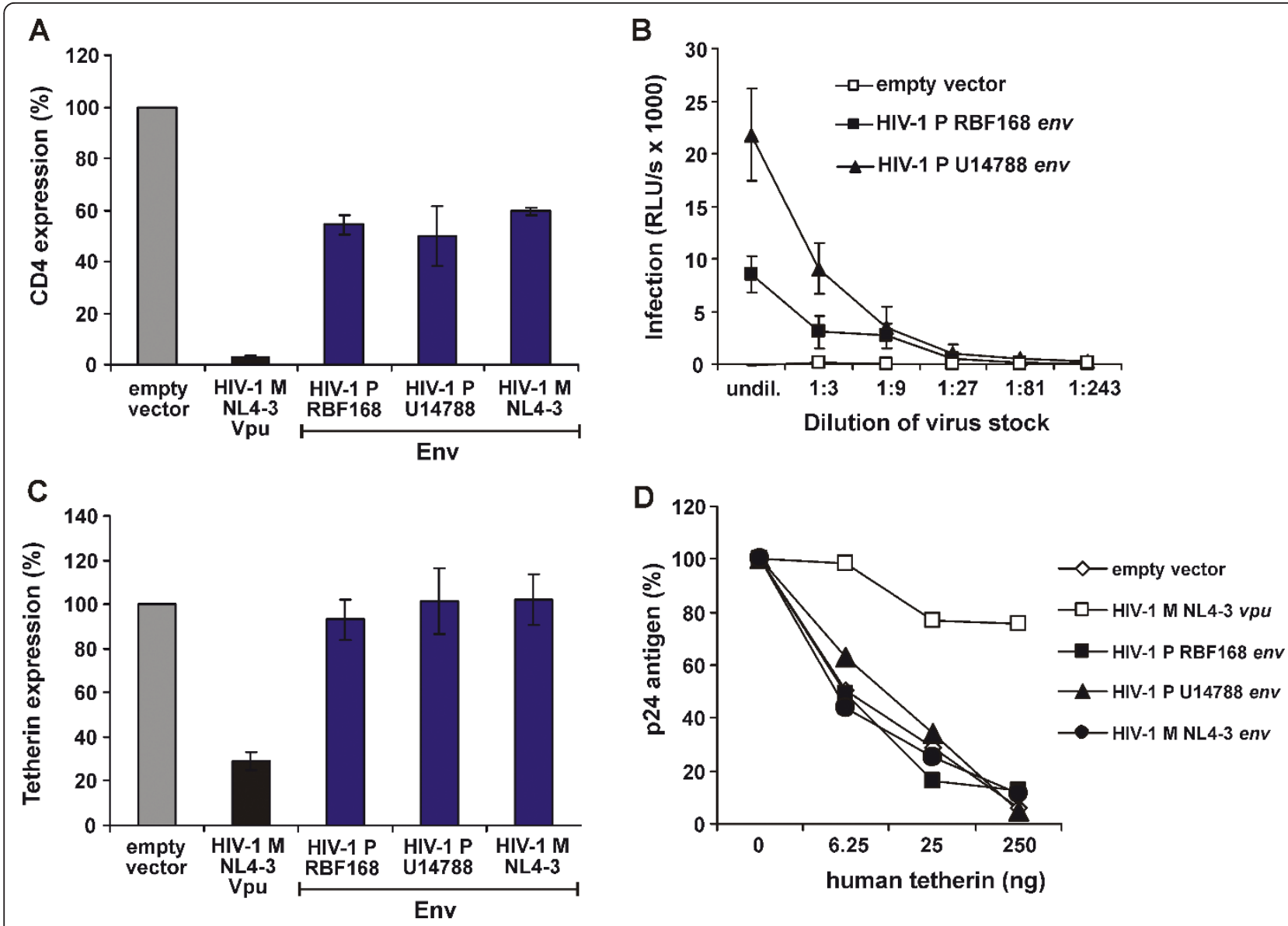

Figure 4 Tetherin Counteraction by HIV-1 Group P Env. (A) FACS analysis of 293T cells cotransfected with a CD4 expression vector and pCAGGS plasmids expressing the indicated env alleles or an empty vector control. Shown are the cell surface expression levels of CD4 relative to those measured in cells transfected with the vector control. The mean $( \pm$ SD) of two independent experiments is shown. (B) Infectivity of virions pseudotyped with HIV-1 P Env. TZM-bl reporter cells were infected with different dilutions of the supernatant of 293T cells cotransfected with a vpu-, nef- and env-deficient HIV-1 NL4-3 proviral construct and the indicated env alleles. Infection was performed in triplicates and results were confirmed in three additional experiments. (C) FACS analysis of 293T cells cotransfected with a human tetherin expression vector and pCAGGS plasmids expressing the indicated env alleles or an empty vector control. Shown are the cell surface expression levels of tetherin relative to those measured in cells transfected with the vector control. The mean ( \pm SD) of two independent experiments is shown. (D) p24 release from 293T cells following transfection with a vpu-, env- and nef-defective proviral HIV-1 NL4-3 construct, pCAGGS plasmids expressing the indicated env alleles and different amounts of human tetherin expression plasmids. p24 release was quantified by ELISA. Values are shown as percentage of values obtained in the absence of tetherin. The mean of two independent experiments is shown.

human tetherin and the rare Group $\mathrm{N}$ viruses gained only modest anti-tetherin activity and lost the second major function of $\mathrm{Vpu}$, i.e. degradation of $\mathrm{CD} 4$, during adaptation to humans $[17,21]$. The recent description of a fourth group of HIV-1, designated "P", that most likely represents an independent cross-species transmission of SIVgor infecting gorillas to humans [22,23], allowed us to further investigate a possible role of effective tetherin antagonism in the spread of the different groups of HIV-1. In the present study, we thus determined whether or not HIV-1 Group P has cleared the tetherin barrier in humans.

To assess whether the transmission event that resulted in HIV-1 Group P also occurred relatively recently, as previously described for Group $\mathrm{M}, \mathrm{O}$ and $\mathrm{N}$ viruses [27-29], we first performed extensive phylogenetic analyses. Our results suggest that Group P entered the human population between 1845 and 1989. This window is rather wide due to the fact that only two HIV-1 Group P strains have been described to date $[22,23]$ and could be further narrowed once additional Group P or SIVgor sequences become available. It is also noteworthy that the possibility that the Group P zoonosis did not result from a gorilla, but from a yet-to-be-identified chimpanzee population can currently not be entirely dismissed [22].

In spite of the aforementioned limitations, our analyses suggest that the transmission of SIVgor that gave rise to HIV-1 Group P occurred within the last 170 years and is 
thus similar to three zoonotic transmission events that led to the emergence of HIV-1 Groups M, O and N [27-29]. Our functional analyses demonstrate that the vpu and nef genes of both HIV-1 Group P strains are unable to antagonize human tetherin. These results are in agreement with a recent study reporting lack of antitetherin activity of Group P Vpu and Nef proteins [38], published while this manuscript was under revision. Our analyses significantly expand these findings because only the RBF168 strain was examined in the previous study, whereas we also examined the Vpu and Nef proteins of the second HIV-1 Group P 06CMU14788 strain. Perhaps even more importantly, we also show that the Env proteins of both HIV-1 Group P strains do not antagonize tetherin, although they are otherwise active and capable of mediating HIV-1 entry. These results strongly suggest that HIV-1 Group P has not yet evolved an effective antagonist of human tetherin. A recent origin and suboptimal adaptation to the new human host is further supported by the fact that only one of the two HIV-1 Group $\mathrm{P}$ strains (06CMU14788) has evolved a human-specific change of $\mathrm{M} 30 \mathrm{~K}$ in the viral $\mathrm{p} 17$ matrix protein that is present in all other groups of HIV-1 and seems to be relevant for the replicative fitness of HIV-1 in the human host [39].

We found that the HIV-1 Group P Nef was unable to promote virion release although it reduced the levels of cell surface expression of the gorilla tetherin orthologue by up to 2 -fold. This result is in agreement with recent data on $\mathrm{Vpu}$ function showing that promotion of virus release and downmodulation of tetherin from the cell surface do not always correlate $[35,36]$. A possible reason for this is that effective tetherin antagonism by $\mathrm{Vpu}$ and/or Nef may involve several distinct but cooperative mechanisms $[16,40]$. For $\mathrm{Vpu}$, it has been reported that it interacts directly with the transmembrane domain of tetherin, sequesters the restriction factor away from the sites of viral budding to the trans-Golgi network and the perinuclear compartment and induces its proteasomal and/or lysosomal degradation [41-43]. The mechanism of Nefmediated tetherin antagonism is poorly investigated and may involve AP-2 dependent removal of tetherin from the sites of virion assembly [44]. It is tempting to speculate that some Vpu and Nef proteins may be able to downmodulate tetherin from the cell surface and/or to increase its degradation but lack the capability to sequester tetherin away from the sites of virion budding and are thus unable to efficiently promote the release of progeny virions.

\section{Conclusions}

In conclusion, our data further support that effective tetherin antagonism may play a role in the effective spread of HIV/AIDS. However, further studies using replication-competent full-length molecular clones of
HIV-1 Group O, N and P strains are required to exclude the possibility that they may counteract the tetherin restriction through an as yet unknown mechanism. It is evident that HIV-1 Groups $\mathrm{O}$ and $\mathrm{N}$ can cause AIDS $[45,46]$, whereas the pathogenic potential of Group P remains to be determined. Furthermore, it remains elusive whether the viral loads, rates of CD4 T cell decline and clinical progression, or the efficiency of virion shedding into genital fluids, differ between individuals infected with pandemic HIV-1 Group M and non-pandemic Group O or rare Group N and P strains. In-depth studies of the virological, immunological and clinical characteristics of Group O, N and P infections in humans may provide important insights into virus transmission and seem highly warranted.

\section{Methods \\ Dating the most recent common ancestor of HIV-1 Group $P$ and SIVgor}

Full-length genome sequences representative of HIV-1 and related SIV strains were retrieved from Genbank, together with the corresponding sampling dates. The dataset included four SIVcpz sequences from Pan troglodytes schweinfurthii (GenBank accession numbers: :AF447763; EF394357; EF394358; U42720), ten SIVcpz sequences from Pan troglodytes troglodytes (AF103818; AF115393; AJ271369; AY169968; DQ373063; DQ373064; DQ373065; DQ373066; EF535994; X52154), four SIVgor sequences from Gorilla gorilla gorilla (FJ424863; FJ424864; FJ424865; FJ424871); eight reference HIV-1 Group M sequences from subtype A (AB253429), subtype B (K03455), subtype C (U46016), subtype D (K03454), subtype F (AJ249238), subtype H (AF190127), subtype J (AF082394) and subtype K (AJ249235); four HIV-1 Group N sequences (AJ006022; AJ271370; AY532635; DQ017383); four HIV-1 Group O sequences (AJ302647; AY169812; L20571; L20587); two HIV-1 Group P sequences available to date (GQ328744; HQ179987). A multiple nucleotide sequence alignment was created using the program MUSCLE version 3.6 http://www.drive5.com/muscle/download3.6.html, and manually edited with the program Se-Al http://tree.bio.ed. ac.uk/software/seal/. Regions of the alignment that could not be unambiguously aligned where excluded from the analysis. Dated phylogenies and time estimates were initially obtained for the gag, pol and env loci under various evolutionary and demographic assumptions.

The full-length, gag, pol, and env sequence alignments were used to reconstruct the phylogenies of the selected viruses using a Bayesian Markov chain Monte Carlo (MCMC) framework. A posterior distribution of phylogenetic trees was obtained for each alignment using the software MrBayes version 3.1.2 http://mrbayes.csit.fsu.edu/ download.php, under the General Time Reversible model of nucleotide substitution with proportion of invariable 
sites and gamma-distributed rate heterogeneity $(G T R+I+\Gamma)$. The MCMC search was set to $10,000,000$ iterations, with trees sampled every $1000^{\text {th }}$ generation. Convergence of the estimates was checked using the program Tracer (i.e. effective sampling size $>200$ ). A model incorporating the SRD model for nucleotide substitution, where the evolution of $3^{\text {rd }}$ codon positions is modeled independently to $1^{\text {st }}$ and 2 nd codon positions, a relaxed molecular clock model [47] and a Bayesian Skyline coalescent prior [48] fitted the data significantly better than alternative models (Bayes factor $>20$ ).

\section{Cloning and viral constructs}

RBF168 and 06CMU14788 Vpu (accession nos: GQ328744 and HQ179987) and Nef (accession nos: GQ328744 and HQ179987) were synthesized by Genscript, C-terminally tagged with an AU1-tag and cloned into the pCGCG vector coexpressing eGFP via an IRES [17]. RBF168 and 06CMU14788 Envelopes were also synthesized by Genscript and cloned into the pCAGGS vector as previously described [11]. Human and gorilla tetherin were cloned into the PCGCG vector coexpressing DsRed2. All experiments were performed in transfected 293T that overexpress tetherin, GFP and Nef, Vpu or Env after transfection with the CMV- or chicken $\beta$-actin promoter based pCGCG or pCAGGS expression constructs. These plasmids have been previously described $[17,32,49]$.

\section{CD4 cell surface staining and flow cytometry}

293T cells were cotransfected with human CD4 $(0.5 \mu \mathrm{g})$ and different $v p u$, nef or env alleles $(2.5 \mu \mathrm{g})$ or GFP only. $48 \mathrm{~h}$ hours post transfection CD4 surface expression was measured by flow cytometry as previously described $[17,32]$.

\section{Viral release assay, p24 ELISA, and Western blotting}

$293 \mathrm{~T}$ cells were cotransfected with pBR-NL43 $\Delta n e f \Delta v p u$ $(2.0 \mu \mathrm{g})$, different amounts of tetherin (0-125 ng) and different $v p u$ or $n e f$ alleles $(0.5 \mu \mathrm{g}) .48$ hours post infection virus release was determined by infection of TZM-bl reporter cells. Values were normalized (no tetherin $=100 \%$ ) [17]. p24 ELISA and Western blot were carried out as previously described $[17,32]$. For the verification of activity of P Group Env a pBR-NL43 $\Delta n e f \Delta v p u \Delta e n v$ expression vector was used. TZM-bl reporter cells were used to analyze infectivity of HIV-1 Group P Env pseudotyped virions (NIH AIDS Research and Reference Reagent Program).

\section{Additional material}

Additional file 1: Alignment of SIVgor, HIV-1 $\mathrm{M}$ and P Vpus. Amino acid alignment of Vpu proteins from HIV-1 Group M, Group P and SIVgor. An AxxxAxxxW transmembrane motif that has been shown to be crucial for tetherin antagonism by HIV-1 Group M Vpus is highlighted in yellow.
Additional file 2: Tetherin Counteraction by HIV-1 Group P Vpu and Nef. (A) Infectious virus and (B) p24 release from 293T cells following cotransfection with a vpu- and nef-defective proviral HIV-1 NL4-3 construct, pCGCG plasmids expressing eGFP alone (eGFP only) or together with the indicated vpu or nef alleles and a human or gorilla tetherin expression plasmid. Infectious virus was determined by infection of TZM-bl reporter cells and p24 release was quantified by ELISA. Values are shown as percentage of values obtained in the absence of tetherin. All infections were performed in triplicates. The mean of two independent experiments is shown.

\section{Acknowledgements}

The following reagent was obtained through the NIH AIDS Research and Reference Reagent Program, Division of AIDS, NIAID, NIH: TZM-bl from Dr. John C. Kappes, Dr. Xiaoyun Wu and Tranzyme Inc. This work was supported by Wellcome Trust fellowships to RKG (WT081772MA) and GJT (WT076608), Medical Research Council funding to the MRC Centre for Molecular Virology, the European Union, the NIHR UCLH/UCL Comprehensive Biomedical Research Centre and the Deutsche Forschungsgemeinschaft (DFG).

\section{Author details}

${ }^{1}$ Institute of Molecular Virology, Ulm University Medical Center, 89069, Ulm, Germany. ${ }^{2}$ Division of Infection and Immunity, University College London, Gower Street, London, WC1E 6BT, UK. ${ }^{3}$ Laboratoire associé au Centre National de Référence du Virus de l'Immunodéficience Humaine, Centre Hospitalier Universitaire de Rouen, Rouen, France.

\section{Authors' contributions}

DS participated in the design of the study, performed most of the experiments and contributed to the final manuscript. SH coordinated the phylogenetic studies. SJP performed some of the experiments. JCP and GJT helped to conceive the study and participated in the review of the manuscript. RKG conceived and coordinated the study, carried out the phylogenetic analyses and wrote the draft manuscript. FK participated in the design and coordination of the study and wrote the final manuscript. All authors read and approved the final manuscript

\section{Competing interests}

The authors declare that they have no competing interests.

Received: 17 November 2010 Accepted: 15 December 2011 Published: 15 December 2011

\section{References}

1. Harris RS, Liddament MT: Retroviral restriction by APOBEC proteins. Nat Rev Immunol 2004, 4:868-877.

2. Sheehy AM, Gaddis NC, Choi JD, Malim MH: Isolation of a human gene that inhibits HIV-1 infection and is suppressed by the viral Vif protein. Nature 2002, 418:646-650.

3. Sheehy AM, Gaddis NC, Malim MH: The antiretroviral enzyme APOBEC3G is degraded by the proteasome in response to HIV-1 Vif. Nat Med 2003, 9:1404-1407.

4. Song B: TRIM5alpha. Curr Top Microbiol Immunol 2009, 339:47-66.

5. Kupzig S, Korolchuk V, Rollason R, Sugden A, Wilde A, Banting G: Bst-2/ HM1.24 is a raft-associated apical membrane protein with an unusual topology. Traffic 2003, 4:694-709.

6. Neil SJ, Eastman SW, Jouvenet N, Bieniasz PD: HIV-1 Vpu promotes release and prevents endocytosis of nascent retrovirus particles from the plasma membrane. PLoS Pathog 2006, 2:e39.

7. Neil SJ, Sandrin V, Sundquist WI, Bieniasz PD: An interferon-alpha-induced tethering mechanism inhibits HIV-1 and Ebola virus particle release but is counteracted by the HIV-1 Vpu protein. Cell Host Microbe 2007, 2:193-203.

8. Neil SJ, Zang T, Bieniasz PD: Tetherin inhibits retrovirus release and is antagonized by HIV-1 Vpu. Nature 2008, 451:425-430.

9. Van Damme N, Goff D, Katsura C, Jorgenson RL, Mitchell R, Johnson MC Stephens EB, Guatelli J: The Interferon-Induced Protein BST-2 Restricts 
HIV-1 Release and Is Downregulated from the Cell Surface by the Viral Vpu Protein. Cell Host Microbe 2008, 3:245-252.

10. Gupta RK, Hue S, Schaller T, Verschoor E, Pillay D, Towers GJ: Mutation of a single residue renders human tetherin resistant to HIV-1 Vpu-mediated depletion. PLoS Pathog 2009, 5:e1000443.

11. Gupta RK, Mlcochova P, Pelchen-Matthews A, Petit SJ, Mattiuzzo G, Pillay D, Takeuchi Y, Marsh M, Towers GJ: Simian immunodeficiency virus envelope glycoprotein counteracts tetherin/BST-2/CD317 by intracellular sequestration. Proc Natl Acad Sci USA 2009, 106:20889-94.

12. Huthoff $H$, Malim MH: Identification of amino acid residues in APOBEC3G required for regulation by human immunodeficiency virus type 1 Vif and Virion encapsidation. J Virol 2007, 81:3807-3815.

13. Yap MW, Nisole S, Stoye JP: A Single Amino Acid Change in the SPRY Domain of Human Trim5alpha Leads to HIV-1 Restriction. Curr Biol 2005, 15:73-78.

14. Hahn BH, Sharp PM: The evolution of HIV-1 and the origin of AIDS. Philos Trans R Soc Lond B Biol Sci 2010, 365:2487-94.

15. Jia B, Serra-Moreno R, Neidermyer W, Rahmberg A, Mackey J, Fofana IB, Johnson WE, Westmoreland S, Evans DT: Species-specific activity of SIV Nef and HIV-1 Vpu in overcoming restriction by tetherin/BST2. PLOS Pathog 2009, 5:e1000429.

16. Sauter D, Kirchhoff F: Tetherin Antagonism by Primate Lentiviral Nef Proteins. Curr HIV Res 2011.

17. Sauter D, Schindler M, Specht A, Landford WN, Munch J, Kim KA, Votteler J, Schubert U, Bibollet-Ruche F, Keele BF, Takehisa J, Ogando Y,

Ochsenbauer C, Kappes JC, Ayouba A, Peeters M, Learn GH, Shaw G, Sharp PM, Bieniasz P, Hahn BH, Hatziioannou T, Kirchhoff F: Tetherin-driven adaptation of Vpu and Nef function and the evolution of pandemic and nonpandemic HIV-1 strains. Cell Host Microbe 2009, 6:409-421.

18. Sauter D, Specht A, Kirchhoff F: Tetherin: holding on and letting go. Cell 2010, 141:392-398.

19. Schmökel J, Sauter D, Schindler M, Leendertz FH, Bailes E, Dazza MC, Saragosti S, Bibollet-Ruche F, Peeters M, Hahn BH, Kirchhoff F: The presence of a vpu gene and the lack of Nef-mediated downmodulation of T cell receptor-CD3 are not always linked in primate lentiviruses. J Virol 2011, 85:742-52

20. Zhang F, Wilson SJ, Landford WC, Virgen B, Gregory D, Johnson MC, Munch J, Kirchhoff F, Bieniasz PD, Hatziiannou T: Nef proteins from simian immunodeficiency viruses are tetherin antagonists. Cell Host Microbe 2009, 6:54-67.

21. Yang SJ, Lopez LA, Hauser H, Exline CM, Haworth KG, Cannon PM: Anti-tetherin activities in Vpu-expressing primate lentiviruses. Retrovirology 2010, 7:13.

22. Plantier JC, Leoz M, Dickerson JE, De Oliveira F, Cordonnier F, Lemee V, Damond F, Robertson DL, Simon F: A new human immunodeficiency virus derived from gorillas. Nat Med 2009, 15:871-872.

23. Vallari A, Yamaguchi J, Holzmayer V, Harris B, Ngansop C, Mbanya D, Ndembi N, Kaptue L, Devare S, Brennan C: HIV-1 Group P: confirmation of group P in Cameroon, 17th Conference on Retroviruses and Opportunistic Infections, San Francisco. 2010.

24. Drummond AJ, Nicholls GK, Rodrigo AG, Solomon W: Estimating mutation parameters, population history and genealogy simultaneously from temporally spaced sequence data. Genetics 2002, 161:1307-1320.

25. Drummond AJ, Rambaut A: BEAST: Bayesian evolutionary analysis by sampling trees. BMC Evol Biol 2007, 7:214

26. Takehisa J, Kraus MH, Ayouba A, Bailes E, Van Heuverswyn F, Decker JM, Li Y, Rudicell RS, Learn GH, Neel C, Ngole EM, Shaw GM, Peeters M, Sharp PM, Hahn BH: Origin and biology of simian immunodeficiency virus in wild-living western gorillas. Journal of Virology 2009, 83:1635-1648.

27. Lemey P, Pybus OG, Rambaut A, Drummond AJ, Robertson DL, Roques $P$, Worobey M, Vandamme AM: The molecular population genetics of HIV-1 group O. Genetics 2004, 167:1059-1068.

28. Wertheim JO, Worobey M: Dating the age of the SIV lineages that gave rise to HIV-1 and HIV-2. PLoS Comput Biol 2009, 5:e1000377.

29. Worobey M, Gemmel M, Teuwen DE, Haselkorn T, Kunstman K, Bunce M, Muyembe JJ, Kabongo JMM, Kalengayi RM, EV M, Gilbert MTG, Wolinsky SM: Direct evidence of extensive diversity of HIV-1 in Kinshasa by 1960. Nature 2008, 455:661-664

30. Greenberg ME, Bronson S, Lock M, Neumann M, Pavlakis GN, Skowronski J: Co-localization of HIV-1 Nef with the AP-2 adaptor protein complex correlates with Nef-induced CD4 down-regulation. Embo J 1997, 16:6964-6976.
31. Petit SJ, Blondeau C, Towers GJ: Analysis of the human immunodeficiency virus type $1 \mathrm{M}$ group Vpu domains involved in antagonizing tetherin. J Gen Virol 2011, 92:2937-48.

32. Schindler M, Munch J, Kutsch O, Li H, Santiago ML, Bibollet-Ruche F, MullerTrutwin MC, Novembre FJ, Peeters M, Courgnaud V, Bailes E, Roques $P$, Sodora DL, Silvestri G, Sharp PM, Hahn BH, Kirchhoff F: Nef-mediated suppression of $\mathrm{T}$ cell activation was lost in a lentiviral lineage that gave rise to HIV-1. Cell 2006, 125:1055-1067.

33. Rucker E, Grivel JC, Munch J, Kirchhoff F, Margolis L: Vpr and Vpu are important for efficient human immunodeficiency virus type 1 replication and CD4+ T-cell depletion in human lymphoid tissue ex vivo. J Virol 2004, 78:12689-12693.

34. Vigan $\mathrm{R}$, Neil SJ: Determinants of tetherin antagonism in the transmembrane domain of the human immunodeficiency virus type 1 Vpu protein. J Virol 2010, 84:12958-70.

35. Goffinet C, Homann S, Ambiel I, Tibroni N, Rupp D, Keppler OT, Fackler OT: Antagonism of CD317 restriction of human immunodeficiency virus type 1 (HIV-1) particle release and depletion of CD317 are separable activities of HIV-1 Vpu. J Virol 2010, 84:4089-94.

36. Kuhl BD, Sloan RD, Donahue DA, Liang C, Wainberg MA: Vpu-mediated tetherin antagonism of ongoing HIV-1 infection in CD4(+) T-cells is not directly related to the extent of tetherin cell surface downmodulation. Virology 2011, 417:353-61.

37. Le Tortorec A, Neil SJ: Antagonism to and intracellular sequestration of human tetherin by the human immunodeficiency virus type 2 envelope glycoprotein. J Virol 2009, 83:11966-11978.

38. Yang SJ, Lopez LA, Exline CM, Haworth KG, Cannon PM: Lack of adaptation to human tetherin in HIV-1 group O and P. Retrovirology 2011, 8:78.

39. Wain LV, Bailes E, Bibollet-Ruche F, Decker JM, Keele BF, van Heuverswyn F, Li Y, Takehisa J, Ngole EM, Shaw GM, Peeters M, Hahn BH, Sharp PM: Adaptation of HIV-1 to its human host. Mol Biol Evol 2007, 24:1853-60.

40. Dubé M, Roy BB, Guiot-Guillain P, Binette J, Mercier J, Chiasson A, Cohen EA: Antagonism of tetherin restriction of HIV-1 release by Vpu involves binding and sequestration of the restriction factor in a perinuclear compartment. PLOS Pathog 2010, 6:e1000856.

41. Goffinet C, Allespach I, Homann S, Tervo HM, Habermann A, Rupp D, Oberbremer L, Kern C, Tibroni N, Welsch S, Krijnse-Locker J, Banting G, Kräusslich HG, Fackler OT, Keppler OT: HIV-1 Antagonism of CD317 is Species Specific and Involves Vpu-Mediated Proteasomal Degradation of the Restriction Factor. Cell Host Microbe 2009, 5:285-97.

42. Mangeat B, Gers-Huber G, Lehmann M, Zufferey M, Luban J, Piguet V: HIV-1 Vpu neutralizes the antiviral factor Tetherin/BST-2 by binding it and directing its beta-TrCP2-dependent degradation. PLoS Pathog 2009, 5:1000574

43. Mitchell RS, Katsura C, Skasko MA, Fitzpatrick K, Lau D, Ruiz A, Stephens EB, Margottin-Goguet F, Benarous R, Guatelli JC: Vpu antagonizes BST-2mediated restriction of HIV-1 release via beta-TrCP and endo-lysosomal trafficking. PLoS Pathog 2009, 5:e1000450.

44. Zhang F, Landford WM, Ng M, MCNatt MW, Bieniasz PD, Hatziiannou T: SIV Nef proteins recruit the AP-2 complex to antagonize Tetherin and facilitate virion release. PLoS Pathog 2011, 7:e1002039.

45. Lindboe CF, Frøland SS, Wefring KW, Linnestad PJ, Bøhmer T, Foerster A, Løken AC: Autopsy findings in three family members with a presumably acquired immunodeficiency syndrome of unknown etiology. Acta Pathol Microbiol Immunol Scand A 1986, 94:117-23.

46. Simon F, Mauclère $P$, Roques $P$, Loussert-Ajaka I, Müller-Trutwin MC, Saragosti $S$, Georges-Courbot MC, Barré-Sinoussi F, Brun-Vézinet F: Identification of a new human immunodeficiency virus type 1 distinct from group M and group O. Nat Med 1998, 4:1032-7.

47. Drummond AJ, Ho SY, Phillips MJ, Rambaut A: Relaxed phylogenetics and dating with confidence. PLOS Biol 2006, 4:e88

48. Drummond AJ, Rambaut A, Shapiro B, Pybus OG: Bayesian coalescent inference of past population dynamics from molecular sequences. $\mathrm{Mol}$ Biol Evol 2005, 22:1185-1192.

49. Tanaka M, Herr W: Differential transcriptional activation by Oct-1 and Oct-2: interdependent activation domains induce Oct-2 phosphorylation. Cell 1990, 60:375-386.

doi:10.1186/1742-4690-8-103

Cite this article as: Sauter et al: HIV-1 Group P is unable to antagonize human tetherin by Vpu, Env or Nef. Retrovirology 2011 8:103. 